\title{
Hybrid teaching workshops: upskilling educators to deliver hybrid classes
}

\author{
Sandris Zeivots \\ University of Sydney, Australia \\ Courtney Shalavin \\ University of Sydney, Australia
}

Keywords: hybrid teaching; hybrid classes; academic professional development; Covid19; pandemic pedagogy.

\section{The challenge}

The Covid-19 pandemic has fundamentally challenged teaching and learning practices in higher education. In early 2020, the University of Sydney Business School joined many other institutions in rapidly pivoting to online teaching in response to social distancing requirements. While online and blended delivery modes were practised pre-pandemic, more traditional face-to-face teaching methods were the 'norm'. The School's educators rose to the challenge of delivering most classes online by the end of year. In early 2021, continually changing strategic and financial circumstances posed a new challenge - some courses required delivery in a hybrid mode. Hybrid teaching requires an educator to manage on-campus and remote students simultaneously (Baker et al., 2020). The majority of staff at the Business School had limited experience and understanding of hybrid delivery.

The Business Co-Design (BCD) team, a mix of educational developers, learning designers, and learning technologists, was approached to meet the challenge of preparing academic staff for teaching in hybrid mode. Two of us - an educational developer and a learning designer from $B C D$ - were tasked with delivering experiential workshops on hybrid teaching that were tailored to the needs of our academic colleagues in the Business School. 


\section{The response}

We facilitated six experiential workshops to inform and prepare educators for hybrid teaching. The aim of the workshops was four-fold: to inform educators of good practice in hybrid teaching; to support educators in the planning and delivery of hybrid classes; to introduce educators to hybrid teaching spaces available in the Business School; and to provide at-elbow advice on transforming classes into hybrid mode (Cusano, 2018).

The workshops were delivered in hybrid mode to simulate the experience of teaching and participating in hybrid classes. Workshops ran for 45 minutes and leveraged the videoconferencing tool Zoom and available AV technologies at the university to blend the virtual and physical environments. We structured the workshops into three stages: overviewing strategies for hybrid teaching and available support at the university; participating in two experiential hybrid activities using Padlet and Jamboard; and a Q\&A session with an opportunity for at-elbow advice. We considered the practical implications of our design choices, choosing to keep everything, above all else, simple. We avoided overcomplicated definitions of hybrid teaching and technologies that were not widely available. We chose to showcase two collaborative tools - Padlet and Jamboard - that were effective at blending the physical and virtual learning environments together, free to use, straight-forward to navigate and implement, and could be employed to engage students with synchronous learning activities.

Overall, the workshops were well-attended and valuable. A total of 86 educators attended the workshops, with 78 of these participants joining via Zoom. The results of an evaluation survey (12\% participation rate) indicated that the workshops were well-received. The survey explored participants' motivations for attending the workshop, prior knowledge of hybrid teaching, and valuable learning obtained in the workshop. Survey respondents indicated that they had limited knowledge and experience of teaching hybrid classes prior to attending the workshop. The primary motive for participants' workshop attendance, cited by respondents to the survey, was to learn about hybrid teaching classes (70\%), followed by personal development $(50 \%)$, and preparation for the eventuality that they would have to teach a hybrid class (40\%). All respondents indicated that they learnt multiple skills and strategies, with the most popular being tips for hybrid teaching (90\%), how to use interactive tools $(80 \%)$, strategies for teaching hybrid classes $(70 \%)$, and how to overcome 
potential challenges with hybrid teaching (60\%). Valuable learning obtained from the workshop included: useful hints, recommendations that were helpful and well explained, and 'good general guidance'. Survey respondents had concerns about using different tools and spending too much time on technology rather than content and learning, and also wished to learn more about setting up classrooms for hybrid teaching.

\section{Recommendations}

Delivering workshops in hybrid mode was challenging. Workshop participants overwhelmingly chose to attend the workshops online, which in some instances meant that we were delivering to an empty room. It was evident from the survey feedback that some participants were unsure about how to use the physical classroom space in hybrid mode which may have been alleviated if they had attended on campus.

After delivering six hybrid teaching workshops for academic staff, we recommend:

- Encouraging participants to attend face-to-face, if circumstances permit, to ensure they get a feel for how to leverage the physical classroom space in hybrid mode. We observed that in-person attendees particularly benefited from the workshop as they often stayed longer and asked specific questions around the physical learning space, technology, and their individual context. Outlining the benefits of face-to-face participation in workshop invitations and promotions may encourage participants to attend in person.

- Experimenting with workshop delivery to showcase various approaches in hybrid teaching (Richardson et al., 2020). Over six workshops we tested three different audio and visual input and output options including audio and visual input and output through the teacher's laptop, audio and visual input and output through available AV technologies, and a combination of both options. We trialled these modes and discussed the benefits and challenges directly with participants. In some instances, we invited participants to experience poor audio quality to highlight the importance of clear sound quality.

- Keeping the workshops simple and tailored. We noticed that attendees were mostly interested in general enquiries about hybrid teaching in case they are required to deliver hybrid classes in the forthcoming semesters. The spectrum of staff attending 
the workshops varied: from academics with no expectations of teaching hybrid classes to those making specific requests about using Padlet in a hybrid class scheduled a few hours after the workshop. The last 15-minute Q\&A section was essential to address the diverse set of enquiries, concerns, and curiosities about hybrid teaching.

- That universities employ institution-wide definitions of relevant terms. Several enquiries raised a concern about using similar terms such as hybrid, hyflex, blended, and online teaching interchangeably. Institution-wide definitions may avoid confusion and foster consistency.

\section{References}

Baker, D. M. A., Unni, R., Kerr-Sims, S. and Marquis, G. (2020) 'Understanding factors that influence attitude and preference for hybrid course formats', The e-Journal of Business Education \& Scholarship of Teaching, 14(1), pp.174-188.

Cusano, C. (2018) Vocational instructors experience and practice teaching in the hybrid environment. Unpublished PhD thesis. Walden University.

Richardson, J. W., Lingat, J. E. M., Hollis, E. and Pritchard, M. (2020) 'Shifting teaching and learning in online learning spaces: an investigation of a faculty online teaching and learning initiative', Online Learning, 24(1), pp.67-91.

https://doi.org/10.24059/olj.v24i1.1629

\section{Author Details}

Sandris Zeivots is a lecturer in Educational Development at the University of Sydney Business School. He investigates how to design and implement innovative learning experiences that are meaningful, engaging, and purposeful.

Courtney Shalavin is a Learning Designer at the University of Sydney Business School. Her work focuses on technology-enabled work-integrated learning, sustainable learning design, and interactive learning in large classes. 